\title{
PRODUCTION OF A NEW MAGNETIC NANOCOMPOSITE FROM WEEDS BIOCHAR
}

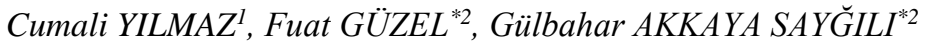 \\ ${ }^{1}$ Department of Chemistry, Institute of Natural and Applied Sciences, Dicle University, 21280 Diyarbakir, Turkey \\ ${ }^{2 *}$ Department of Chemistry, Faculty of Education, Dicle University, 21280 Diyarbakır, Turkey
}

\begin{abstract}
In this study, the synthesis and characterization of a novel composite material was carried out by coprecipitation method. Weeds biochar was successfully transferred to a magnetic material via embedding $\mathrm{MnFe}_{2} \mathrm{O}_{4}$ nanoparticles to its structure. The synthesized spinel ferrite composite was characterized with scanning electron microscopy and vibrating sample magnetometer spectroscopy and the results of the analyses of non-magnetic and magnetic material were compared.
\end{abstract}

Key words: weeds, biochar, magnetic composite, magnetite nanoparticles, characterization

\section{Introduction}

Magnetic ferrite $\mathrm{MFe}_{2} \mathrm{O}_{4}$ (M denotes a divalent metal such as $\mathrm{Fe}^{\mathrm{II}}, \mathrm{Mn}^{\mathrm{II}}, \mathrm{Cu}^{\mathrm{II}}, \mathrm{Zn}^{\mathrm{II}}$ ) nanoparticles (NPs) possess special optical, electrical, and magnetic properties, thus have recently attracted much attention in magnetic storage, biosensing, disease diagnosis, catalysis, and environmental analysis [1]. However preventing NPs aggregation, a base material is needed. Biochar, such as wood charcoals and crop residue derived chars, refer to thecarbon rich residues from pyrolysis or incomplete combustion of biomass [2]. Agricultural wastes or residues are wide available low cost materials to produce biochar as well as biooil and gases [3]. As such, combining the advantages of cheap biochar and magnetic NPs with an industrial friendly production method may be a promising solution of the magnetic biochar nanocomposite. This study aimed to develop a new magnetic biochar composite via a low cost method. Weeds were firstly converted to biochar and $\mathrm{MnFe}_{2} \mathrm{O}_{4} \mathrm{NPs}$ were embedded to the weeds based biochar. The synthesized magnetic material was characterized and compared with its non-magnetic form.

\section{Materials and Method}

\subsection{Preparation of the weeds based biochar}

The weeds were supplied from the campus of Dicle University in Diyarbakır, Turkey, and then washed, dried and sized to $1-2 \mathrm{~cm}$ in length. The preparation of weeds biochar was performed in a horizontal stainless-steel tubular reactor $(7.0 \mathrm{~cm}$ diameter $\mathrm{x} 100 \mathrm{~cm}$ length) under nitrogen atmosphere $(99.99 \%)$ flow $(100 \mathrm{~mL} / \mathrm{min})$ at the rate of $5{ }^{\circ} \mathrm{C} / \mathrm{min}$ at $500{ }^{0} \mathrm{C}$ for $1 \mathrm{~h}$. Subsequently, the char product was cooled to room temperature, washed with hot deionized water and $\mathrm{HCl}$ of $0.1 \mathrm{M}$ until the $\mathrm{pH}$ of the washing solution reached 6-7, and dried at $105{ }^{\circ} \mathrm{C}$ for $12 \mathrm{~h}$ and then sieved between 80 and 40 meshes.

*Corresponding author: Address: Department of Chemistry, Faculty of Education, Dicle University, 21280 Diyarbakır, Turkey. E-mail address: fguzel@dicle.edu.tr, Phone: +904122481000 


\subsection{Preparation of the magnetic composite}

In order to synthesize magnetic composite, co-precipitation method was used as follows: The stoichiometric amounts of $\mathrm{FeCl}_{3}$ and $\mathrm{MnCl}_{2}$ were dissolved in distilled water. A certain amount of weeds biochar was added to the mixture under vigorous stirring. Then $\mathrm{NaOH}$ solution was used to adjust the $\mathrm{pH}$ of the solution to the range of 10-11. After $\mathrm{pH}$ adjustment, the solution was kept at $100{ }^{\circ} \mathrm{C}$ for $4 \mathrm{~h}$. Then, the solution was filtrated, washed with distilled water several times and dried at $105{ }^{\circ} \mathrm{C}$ for $24 \mathrm{~h}$. The synthesized material was stored in glass bottles.

\subsection{Characterization studies}

The surface morphology was identified by the scanning electron microscopy (SEM) instrument (Carl Zeiss Ultra Plus, UK). Micrographs of samples were observed by the assistance of coated with platinum, operating at $10 \mathrm{kV}$.

Vibrating sample magnetometer (VSM 7404, Lake Shore Cryotronics, USA) was utilized to magnetic features of the synthesized material.

\section{Results and Discussion}

\subsection{SEM analysis}

SEM micrographs of weeds, weeds biochar and $\mathrm{MnFe}_{2} \mathrm{O}_{4} /$ weeds biochar composite are indicated in Figure 1. The surfaces of the materials are different from each other and the successfull loading of $\mathrm{MnFe}_{2} \mathrm{O}_{4}$ NPs can be obserevd from SEM images.
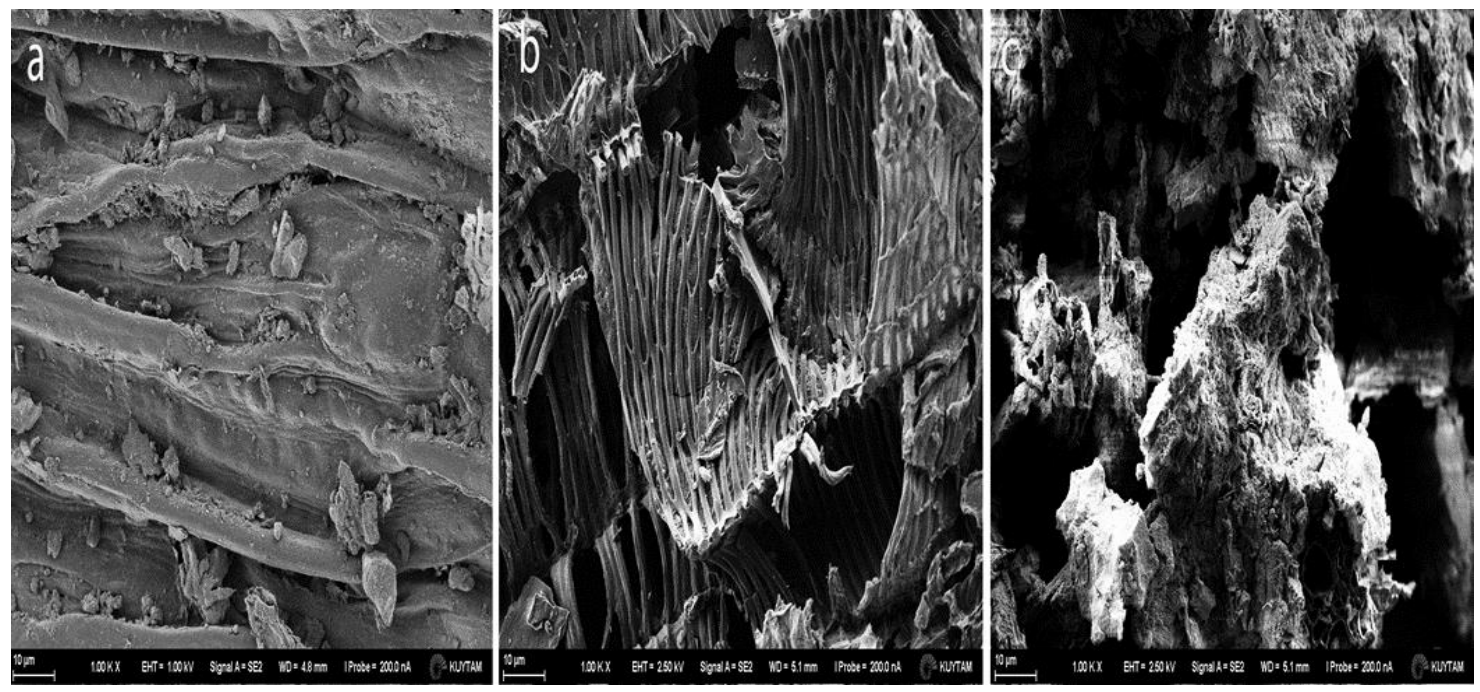

Figure 1. SEM images of weeds (a), weeds biochar (b) and $\mathrm{MnFe}_{2} \mathrm{O}_{4} /$ weeds biochar composite (c).

\subsection{VSM analysis}


VSM curves of the $\mathrm{MnFe}_{2} \mathrm{O}_{4} /$ weeds biochar is seen in Figure 2 and indicates the magnetic property of the synthesized material. Also the inset figure shows that the synthesized composite material is magnetically separable.

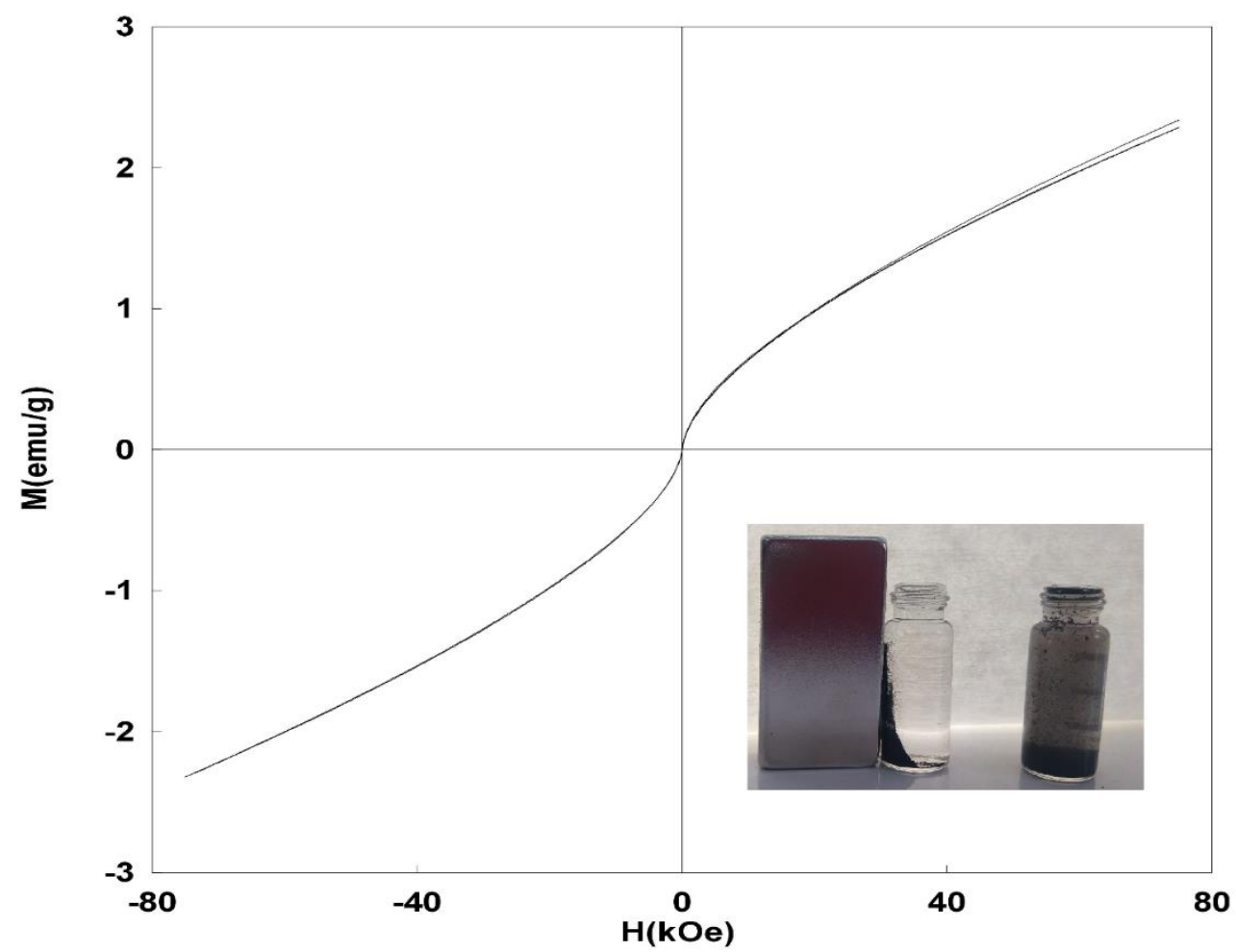

Figure 2. VSM curves of $\mathrm{MnFe}_{2} \mathrm{O}_{4} /$ weeds biochar composite.

\section{Conclusions}

Development of a new magnetic material was achieved from weeds based biochar. The advantages of biochar features were combined with magnetic separabilty and then the functionailty of the biochar was enhanced. This study indicated the possible usage of weeds for the production of magnetic materials. The synthesized material was characterized in terms of surface structure and magnetic properties and the results confirmed the successful embedding of the $\mathrm{MnFe}_{2} \mathrm{O}_{4}$ nanoparticles to the weeds biochar structure.

\section{Acknowledgement}

The authors thank to the Scientific Research Projects Unit of Dicle University (project code: ZGEF-17-024) for the financial support.

\section{References}

[1] Srivastava, M., Ojha, A.K., Chaubey, S., Materny, A., Synthesis and optical 
characterization of nanocrystalline $\mathrm{NiFe}_{2} \mathrm{O}_{4}$ structures. J. Alloys Comp. 481 (2009) 515519.

[2] B. Chen, D. Zhou, L. Zhu, Transitional adsorption and partition of nonpolar and polar aromatic contaminants by biochars of pine needles with different pyrolytic temperatures. Environ. Sci. Technol. 42 (2208) 5147-5143.

[3] H. Zhang, R. Xiao, H. Huang, G. Xiao, Comparison of non-catalytic and catalytic fast pyrolysis of corncob in a fluidized bed reactor, Bioresour. Technol. 100 (2009) 1428-1434. 